Research Article

\title{
Immunological Changes in Peripheral Blood of Ankylosing Spondylitis Patients during Anti-TNF- $\alpha$ Therapy and Their Correlations with Treatment Outcomes
}

\author{
Rongjuan Chen $(\mathbb{D}$, Hongyan Qian, Xiaoqing Yuan, Shiju Chen, Yuan Liu, Bin Wang $\mathbb{D}$, \\ and Guixiu Shi
}

\begin{abstract}
Xiamen Key Laboratory of Rheumatology and Clinical Immunology, The First Affiliated Hospital of Xiamen University,
\end{abstract} Xiamen 361003, China

Correspondence should be addressed to Bin Wang; asrheumw@hotmail.com and Guixiu Shi; gshi@xmu.edu.cn

Rongjuan Chen, Hongyan Qian, and Xiaoqing Yuan contributed equally to this work.

Received 11 May 2021; Accepted 11 September 2021; Published 15 October 2021

Academic Editor: Cinzia Ciccacci

Copyright (C) 2021 Rongjuan Chen et al. This is an open access article distributed under the Creative Commons Attribution License, which permits unrestricted use, distribution, and reproduction in any medium, provided the original work is properly cited.

\begin{abstract}
Tumor necrosis factor- $\alpha$ (TNF- $\alpha$ ) inhibitors are the main types of biological conventional synthetic disease-modifying antirheumatic drugs and have efficacy in treating ankylosing spondylitis (AS) which is not sensitive for nonsteroidal antiinflammatory drug. However, the impact of TNF- $\alpha$ inhibitors on immune cells in patients with AS is still clearly undefined, and the impact of immune cells on treatment response is also largely elusive. This study is aimed at evaluating the longitudinal changes of circulating immune cells after anti-TNF- $\alpha$ therapy and their associations with treatment response in AS patients. Thirty-five AS patients receiving the treatment of anti-TNF- $\alpha$ therapy were included into this prospective observational study. The frequencies of immune cells including Th1, Th2, Th17, regulatory $\mathrm{T}$ cell (Treg), $\mathrm{T}$ follicular helper cell (Tfh), and regulatory B cell (Breg) in the peripheral blood were measured by flow cytometry at baseline and 4 time points after therapy. The difference in the circulating immune cells between responders and nonresponders was compared. This study suggested that anti-TNF- $\alpha$ therapy could significantly reduce circulating proinflammatory immune cells such as Th17 and Tfh, but significantly increased the percentages of circulating Treg and Breg. Moreover, circulating Breg may be a promising predictor of response to anti-TNF- $\alpha$ therapy in AS patients.
\end{abstract}

\section{Introduction}

Ankylosing spondylitis (AS) is a chronic inflammatory rheumatic disease characterized by inflammatory back pain and progressive ankylosing in spine [1]. AS can result in impaired physical functions including disability and obviously reduced life quality [2, 3]. Nonsteroidal anti-inflammatory drug (NSAID) is the main recommended first-line drug for the treatment of AS [4]. However, NSAID is not effective for some AS patients especially for those with later stages, and a large part of AS patients are still poorly controlled in clinical practice [5]. Thus, those AS patients need additional treatment with conventional synthetic disease-modifying antirheumatic drugs (DMARDs) or biological DMARDs [5-7]. Tumor necrosis factor- $\alpha$ (TNF- $\alpha$ ) inhibitors are the main types of biological DMARDs and have a well-established efficacy in treating AS, which has largely revolutionized the treatment of AS in the past two decades [8]. Nevertheless, the treatment response is various involving high risk of infections among some patients such as tuberculosis $[9,10]$. Improvement of AS patients' personalized therapy strategy is an urgent need for the heterogeneity in both the pathogenesis and treatment outcomes $[11,12]$. To improve treatment outcomes, minimize infection risk, and reduce costs, it is critical for clinicians to identify responders to specific biological DMARDs and make adequate therapeutic decisions. 
The roles of T cell subsets in the pathogenesis of AS have been reported in plenty works [13-15], and Th17 cells play a critical pathogenic role in the development of AS $[13,16]$. Apart from Th17, other T cell subsets such as Th1 $[14,17]$ and $\mathrm{T}$ follicular helper cell (Tfh) which is correlated with $\mathrm{B}$ cell subtypes [18-20] are also involved in the pathogenesis of AS. Besides, several studies confirm that B cells participate in the pathogenesis of AS, such as increasing regulatory $B$ cell (Breg) in peripheral blood of AS [21-24]. Nevertheless, the impact of anti-TNF- $\alpha$ therapy on those immune cells in AS patients is still not clearly defined, and the impact of immune cells on treatment response is also largely elusive. To evaluate the longitudinal changes of circulating immune cells after anti-TNF- $\alpha$ therapy and their associations with treatment response in AS patients, we performed a prospective observational study of AS patients receiving anti-TNF- $\alpha$ therapy.

\section{Methods}

2.1. Study Design and Patients. Active AS patients aged 2065 years were recruited in the department of rheumatology in The First Affiliated Hospital of Xiamen University. The patients were recruited prospectively and followed up to 6 months after beginning anti-TNF- $\alpha$ therapy. Inclusion criteria were as follows: (1) patients met the 1984 modified New York classification criteria for AS; (2) without treatment history of biological DMARDs such as anti-TNF agents, anti-IL-17 agents, and anti-IL-6 agents; (3) with a Bath Ankylosing Spondylitis Disease Activity Index (BASDAI) score of no less than 1 ; (4) data of clinical characteristics and laboratory testing analyzed in this study were available; (5) receiving a standard treatment of anti-TNF- $\alpha$ inhibitors; and (6) without obvious infections such as tuberculosis. Exclusion criteria were as follows: (1) AS patients had been treated with biologics such as anti-TNF drugs or anti-IL-6 drugs, (2) patients with a history of spinal or joint surgery, (3) patients with other serious diseases such as cancer or cardiovascular diseases, (4) patients had serious adverse events and discontinued treatment, and (5) data of clinical characteristics and laboratory testing analyzed in the present study were not recorded. A total of 35 AS patients meeting both the inclusion and exclusion criteria were finally included between September 2018 and January 2019. The study was approved by the ethics committee of our hospital, and written informed consent was obtained from included patients.

2.2. Outcome Assessment and Data Collection. The primary endpoint was to achieve an improvement of no less than $50 \%$ in patients at 6 months according to BASDAI. Patients received routine monitoring of disease activity at 5 treatment stages including baseline, 1 month, 2 months, 3 months, and 6 months. Patients with a BASDAI 50\% improvement after 6-month treatment were defined as responders, while those failed to gain a BASDAI 50\% improvement were defined as nonresponders. Other clinical and laboratory parameters such as disease duration, erythrocyte sedimentation rate
TABLE 1: Clinical characteristics of total 35 patients at baseline and after follow-up.

\begin{tabular}{lccc}
\hline Characteristic & At baseline & 6 months & $P$ value \\
\hline ESR (median[Q25-Q75]) & $21(9-34)$ & $4(2-11)$ & $<0.001$ \\
CRP (median[Q25-Q75]) & $6.2(1.9-21.3)$ & $1.6(0.5-3.9)$ & 0.002 \\
ASDAS-CRP & $2.8 \pm 0.8$ & $1.4 \pm 0.8$ & $<0.001$ \\
BASDAI & $4.4 \pm 1.0$ & $1.9 \pm 1.3$ & $<0.001$ \\
\hline
\end{tabular}

AS: ankylosing spondylitis; data were shown as mean \pm SD or median $[\mathrm{Q} 25$ - Q75].

(ESR), and C-reactive protein (CRP) were recorded prospectively at the follow-up visit.

2.3. Sample Collection and PBMC Isolation. Peripheral venous blood was collected from each patient at baseline (before treatment) and 4 follow-up stages after the initiation of anti-TNF treatment ( 1 month, 2 months, 3 months, and 6 months). Serum and plasma were collected for the measurement of liver and renal function parameters. $5 \mathrm{ml}$ peripheral venous blood was used for PBMC isolation with FicollPaque density gradient centrifugation. The isolated PBMCs were stored at $-80^{\circ} \mathrm{C}$ until analysis.

2.4. Flow Cytometry Phenotype. The frequencies of immune cells including Th1, Th2, Th17, regulatory $\mathrm{T}$ cell (Treg), Tfh, and Breg in the peripheral blood were measured by flow cytometry. Briefly, PBMCs were isolated and incubated with PMA (10 ng/ml, eBioscience) and BFA $(10 \mu \mathrm{g} / \mathrm{ml}$, eBioscience) for $4 \mathrm{~h}$ then harvested and washed twice for $30 \mathrm{~min}$. Then, cells were stained with anti-CD4 and anti$\mathrm{CD} 25$ for $30 \mathrm{~min}$ at $4^{\circ} \mathrm{C}$. During the intracellular staining, antibodies against IFN- $\gamma$, IL-4, and IL-17A were according to stain Th1, Th2, and Th17, respectively. Intracellular FoxP3 was also stained, and $\mathrm{CD} 4^{+} \mathrm{FoxP}^{+}$was used to determine Treg. $\mathrm{CD} 19^{+} \mathrm{CD} 24^{\mathrm{High}} \mathrm{CD} 38^{\text {High }}$ cells were determined as Breg. $\mathrm{CD}^{+} \mathrm{PD}^{+} \mathrm{CXCR}^{+}$cells were determined as $\mathrm{Tfh}$. The following anti-human antibodies for surface staining or intracellular staining were used: PE-CY7-anti-CD4, PECY5.5-anti-CD25, FITC-anti-IL-17A, PE-anti-Foxp3, FITC-anti-IFN- $\gamma$, PE-anti-IL-4, FITC-anti-CD19, PE-antiCD24, PE-CY7-anti-CD38, PE-anti-PD1, and FITC-antiCXCR5 (all eBioscience).

2.5. Statistical Analysis. Continuous variables were presented as mean \pm standard deviation (SD) or median with quartiles ( Q25-Q75). Difference between responders and nonresponders was determined using Student's $t$-test or MannWhitney $U$ test. Difference for data at different time points was assessed with paired $t$-test. The roles of immune cells at baseline in predicting treatment response were assessed by receiver operating characteristic (ROC) analysis, and the area under the ROC curve (AUC) was calculated. Statistical analyses were performed with STATA (Version 12.0, StataCorps, Texas, USA). Two-sided $P$ values less than 0.05 were considered statistically significant. 

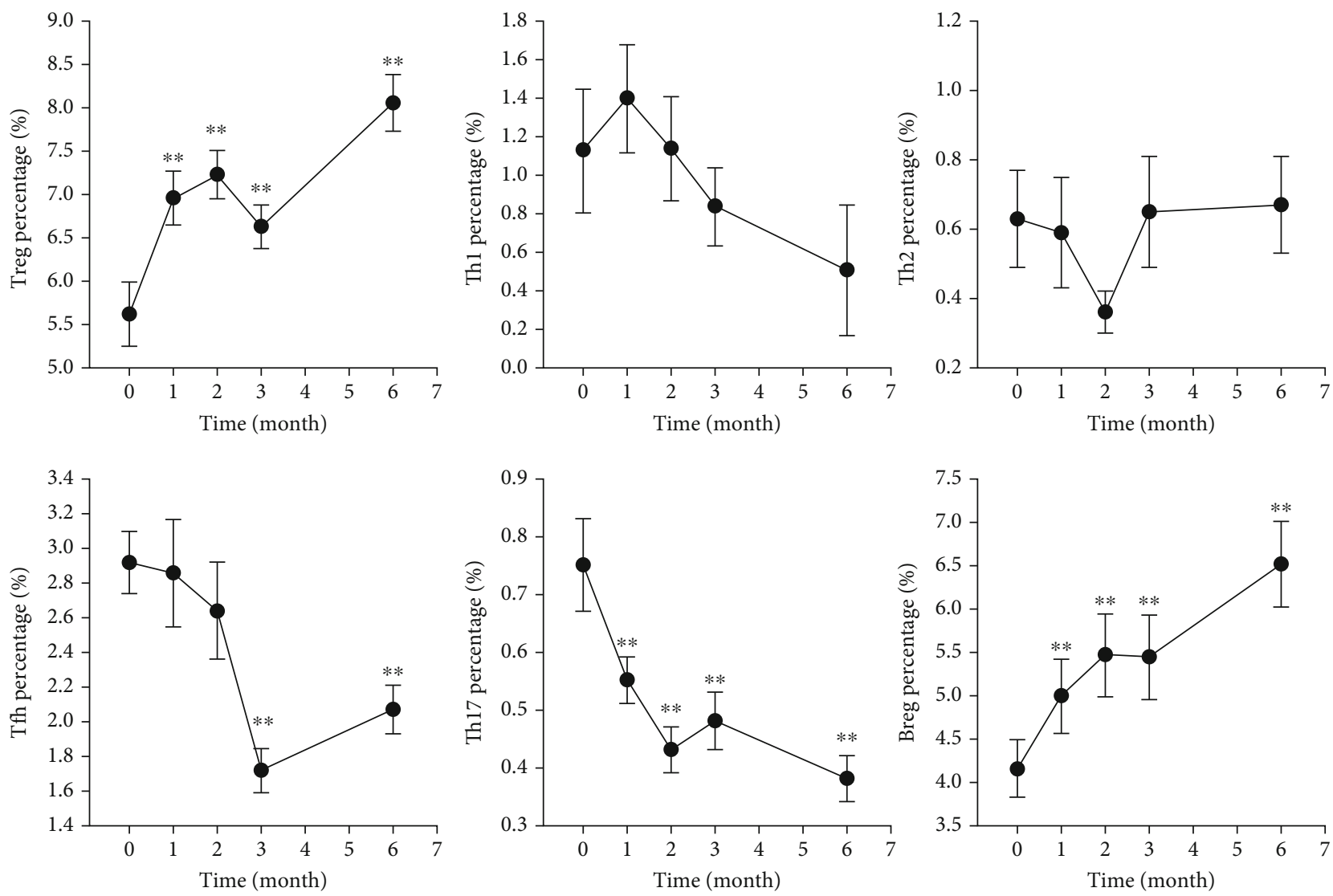

FIGURE 1: Changes of circulating immune cells after anti-TNF- $\alpha$ therapy in AS patients. The percentages of immune cells during follow-up were compared with that at baseline. ${ }^{*} P<0.05 ;{ }^{* *} P<0.005$.

TABLE 2: Changes of immune cells among total 35 patients during follow-up.

\begin{tabular}{lccc}
\hline Immune cells & 0 month & 6 months & $P$ value \\
\hline Treg & $5.62 \pm 2.19$ & $8.06 \pm 1.98$ & $<0.001$ \\
Th17 & $0.75 \pm 0.37$ & $0.38 \pm 0.18$ & $<0.001$ \\
Th17/Treg & $0.15 \pm 0.10$ & $0.05 \pm 0.03$ & $<0.001$ \\
Tfh & $2.92 \pm 0.80$ & $2.07 \pm 0.62$ & $<0.001$ \\
Th1 & $0.30(0.13-1.27)$ & $0.18(0.03-0.25)$ & 0.003 \\
Th2 & $0.38(0.09-0.87)$ & $0.29(0.14-0.89)$ & 0.71 \\
Th1/Th2 & $1.55(0.44-5.33)$ & $0.28(0.10-1.38)$ & $<0.001$ \\
Breg & $4.16 \pm 1.94$ & $6.52 \pm 2.89$ & $<0.001$ \\
CD3 & $73.23 \pm 7.98$ & $67.45 \pm 10.15$ & 0.010 \\
CD4 & $38.04 \pm 5.31$ & $32.25 \pm 7.64$ & $<0.001$ \\
CD8 & $28.49 \pm 8.44$ & $26.22 \pm 8.62$ & 0.27 \\
CD4/CD8 & $1.47 \pm 0.51$ & $1.38 \pm 0.57$ & 0.48 \\
\hline
\end{tabular}

$\overline{\text { Data were shown as mean } \pm \mathrm{SD} \text { [standard deviation] or median [Q25 - Q75] }}$.

\section{Results}

3.1. Clinical Characteristics of AS Patients. Table 1 summarized the clinical and laboratory characteristics of those AS patients (Table 1). Among those 35 AS patients, 30 $(85.7 \%)$ were males. The mean age was $33.1 \pm 8.8$ years old, and the mean disease duration was $8.7 \pm 5.1$ years. At baseline, the mean ASDAS-CRP and BASDAI were $2.8 \pm$ 0.8 and $4.4 \pm 1.0$, respectively. After anti-TNF- $\alpha$ therapy of 6 months, both ESR and CRP were significantly reduced $(P<0.05$; Table 1$)$. The mean ASDAS-CRP significantly declined to $1.4(P<0.001)$ and BASDAI declined to 1.9 $(P<0.001)$ at 6 months. Based on BASDAI, the response rate at 6 months after anti-TNF- $\alpha$ therapy was $60.0 \%$ $(21 / 35)$.

3.2. Changes of Circulating Immune Cells after Anti-TNF- $\alpha$ Therapy. Th1, Th17, and Tfh are common proinflammatory immune cells. After anti-TNF- $\alpha$ therapy, both Th17 and Tfh decreased gradually, and there was also a modest but not significant reduction in Th1 (Figure 1). Anti-TNF- $\alpha$ therapy significantly reduced the percentage of circulating Th17 at 1 month after treatment $(P<0.005)$, and the effect was maintained through treatment course (Figure 1). AntiTNF- $\alpha$ therapy began to significantly reduce the percentage of circulating Tfh at 3 months after treatment $(P<0.005)$, and the effect was also significantly lower at 6 months after treatment $(P<0.005)$. The mean percentage of circulating Th17 significantly decreased from 0.75 to 0.38 after 6 months of anti-TNF- $\alpha$ therapy $(P<0.001$; Table 2$)$.

Th2, Treg, and Breg are key immunoregulatory immune cells. After anti-TNF- $\alpha$ therapy, both Treg and Breg increased gradually, but the frequency of Th2 was not 
TABLE 3: Differences in baseline clinical characteristics and immune cells between responders and nonresponders.

\begin{tabular}{|c|c|c|c|}
\hline Items & Responders $(N=21)$ & Nonresponders $(N=14)$ & $P$ value \\
\hline Gender (male, \%) & $18(85.7 \%)$ & $12(85.7 \%)$ & 1.00 \\
\hline Age (year, mean $\pm S D)$ & $32.86 \pm 7.53$ & $33.43 \pm 10.73$ & 0.85 \\
\hline Disease duration (year, mean $\pm S D$ ) & $8.10 \pm 5.21$ & $9.71 \pm 5.07$ & 0.37 \\
\hline ESR (median[Q25-Q75]) & $13(6-30)$ & $29(14-49)$ & 0.035 \\
\hline CRP (median[Q25-Q75]) & $2.70(1.19-15.72)$ & $8.50(6.65-33.63)$ & 0.018 \\
\hline ASDAS-CRP & $2.73 \pm 0.72$ & $2.98 \pm 0.84$ & 0.35 \\
\hline BASDAI & $4.68 \pm 0.87$ & $3.99 \pm 1.05$ & 0.042 \\
\hline Treg & $5.73 \pm 2.44$ & $5.45 \pm 1.83$ & 0.712 \\
\hline Th17 & $0.76 \pm 0.38$ & $0.74 \pm 0.36$ & 0.880 \\
\hline Th17/Treg & $0.16 \pm 0.10$ & $0.15 \pm 0.11$ & 0.948 \\
\hline Tfh & $2.89 \pm 0.81$ & $2.95 \pm 0.81$ & 0.816 \\
\hline Th1 & $0.18(0.13-0.64)$ & $0.81(0.15-2.62)$ & 0.200 \\
\hline Th2 & $0.43(0.05-0.96)$ & $0.28(0.11-0.74)$ & 0.749 \\
\hline Th1/Th2 & $0.52(0.30-4.26)$ & $2.30(0.71-9.81)$ & 0.178 \\
\hline Breg & $3.62 \pm 1.70$ & $4.97 \pm 2.05$ & 0.041 \\
\hline CD3 & $73.62 \pm 7.87$ & $72.64 \pm 8.40$ & 0.729 \\
\hline $\mathrm{CD} 4$ & $38.02 \pm 5.41$ & $38.08 \pm 5.36$ & 0.976 \\
\hline CD8 & $28.26 \pm 8.07$ & $28.83 \pm 9.27$ & 0.849 \\
\hline $\mathrm{CD} 4 / \mathrm{CD} 8$ & $1.48 \pm 0.54$ & $1.45 \pm 0.46$ & 0.878 \\
\hline
\end{tabular}

Data were shown as mean $\pm \mathrm{SD}$ (standard deviation) or median [Q25 - Q75].

significantly changed (Figure 1). Anti-TNF- $\alpha$ therapy began to significantly increase the percentages of both Treg and Breg at 1 month after treatment $(P<0.005)$. The mean percentage of circulating Treg significantly increased from 5.62 to 8.06 after 6 months of anti-TNF- $\alpha$ therapy $(P<0.001)$, and the mean percentage of circulating Breg significantly increased from 4.16 to $6.52(P<0.001$; Table 2$)$.

3.3. Correlations of Circulating Immune Cells with Response to Anti-TNF- $\alpha$ Therapy in AS Patients. Baseline disease characteristics such as age, disease duration, and ASDAS-CRP were comparable between responders and nonresponders (Table 3). Compared with nonresponders, responders had lower levels of ESR $(P=0.035)$ and CRP $(P=0.018)$ but had higher BASDAI $(P=0.042)$ (Table 3$)$. Compared with those responders, nonresponders had a higher percentage of circulating Breg both at baseline and during follow-up $(P<0.05)$ (Figure 2 and Tables 3 and 4$)$. There was no obvious difference in the baseline percentages of other immune cells such as Th17, Treg, and Tfh between nonresponders and responders (Table 3), and similar findings were also found at 6 months after anti-TNF- $\alpha$ therapy (Table 3).

ROC analysis suggested that Breg was the best circulating cell in predicting response to anti-TNF- $\alpha$ therapy in AS patients (AUC $=0.70,95 \%$ CI 0.52-0.88). Other immune cells had limited roles in predicting response to anti-TNF- $\alpha$ therapy (Figure 3).

\section{Discussion}

The impact of TNF- $\alpha$ inhibitors on immune cells in AS patients is still not clearly defined. Besides, the impact of immune cells on treatment response to TNF- $\alpha$ inhibitors is also largely elusive. This study was thus designed to prospectively evaluate the longitudinal changes of circulating immune cells after anti-TNF- $\alpha$ therapy and their associations with treatment response in AS patients. To our knowledge, this is the first prospective study investigating the impact of immune cells on treatment response to TNF- $\alpha$ inhibitors. We found that both Th17 and Tfh were reduced gradually by anti-TNF- $\alpha$ therapy, while Treg and Breg were increased gradually. Moreover, there was some immunological difference between treatment responders and nonresponders, and responders had a higher percentage of circulating Breg both at baseline and during follow-up, suggesting Breg as a possible predictor of response to anti-TNF$\alpha$ therapy in AS patients.

AS is a heterogeneous disease, and it has been cleared that AS patients have various response to anti-TNF- $\alpha$ therapy [25-27]. BASDAI at baseline has an important impact on assessing the response to therapy in AS [28]. Our work showed that compared with nonresponders, responders had higher BASDAI scores and significant response to anti-TNF- $\alpha$ therapy. This study revealed that about $60 \%$ patients had at least 50\% improvement in BASDAI at 6 months after anti-TNF- $\alpha$ therapy, while the others had poor response. Identification of predictors of treatment outcomes 

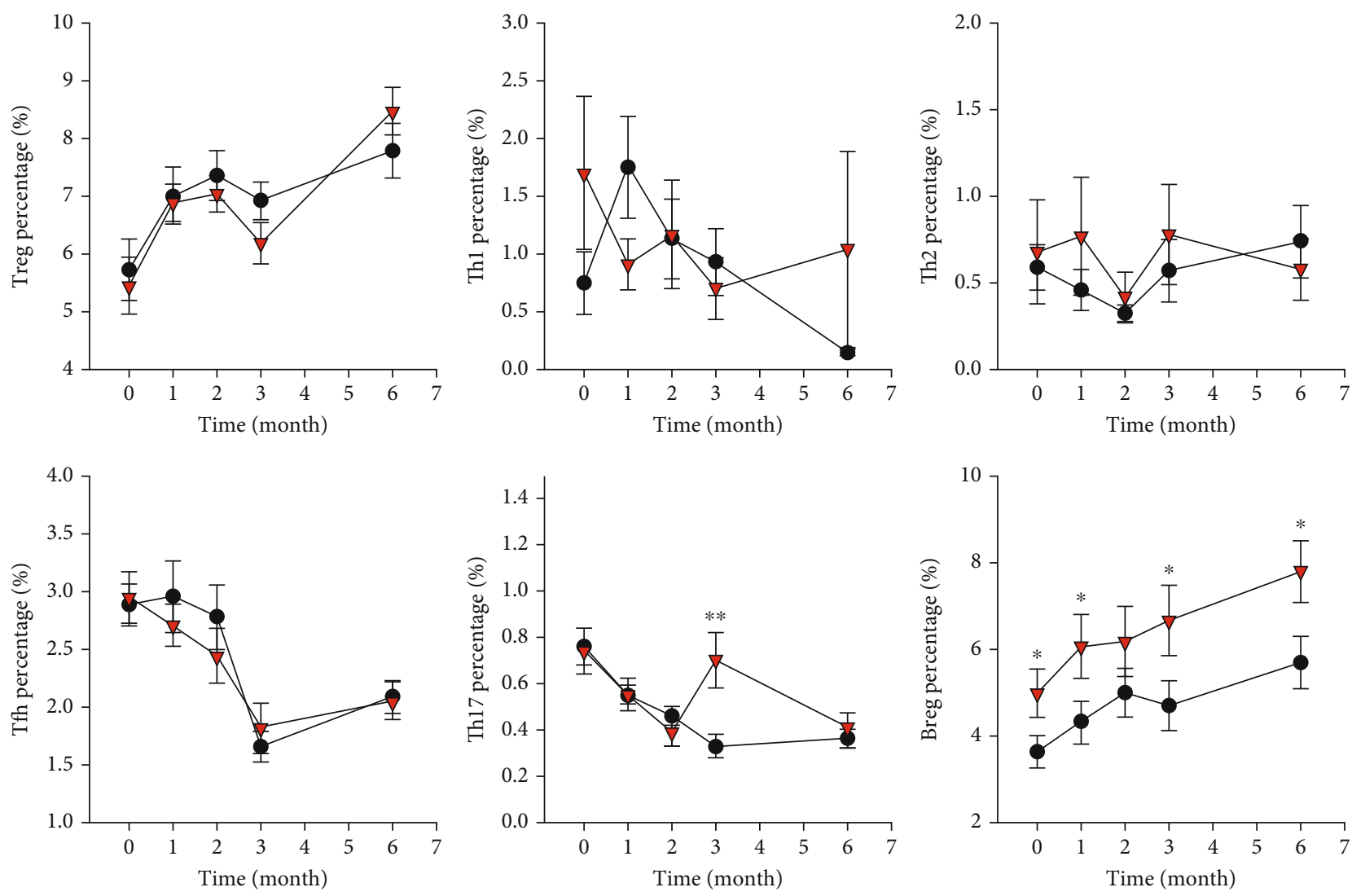

$\rightarrow-$ With response
$\rightarrow-$ No response

FIGURE 2: Changes of circulating immune cells after anti-TNF- $\alpha$ therapy in AS patients stratified by treatment response. Red triangle was for those responders, while black circle was for nonresponders. Difference between responders and nonresponder at each time point was compared. ${ }^{*} P<0.05 ;{ }^{* *} P<0.005$.

TABle 4: Differences in immune cells at 6 months between responders and nonresponders.

\begin{tabular}{lccc}
\hline $\begin{array}{l}\text { Immune } \\
\text { cells }\end{array}$ & $\begin{array}{c}\text { Responders } \\
(N=21)\end{array}$ & $\begin{array}{c}\text { Nonresponders } \\
(N=14)\end{array}$ & $\begin{array}{c}P \\
\text { value }\end{array}$ \\
\hline Treg & $7.79 \pm 2.22$ & $8.47 \pm 1.52$ & 0.323 \\
Th17 & $0.36 \pm 0.16$ & $0.41 \pm 0.21$ & 0.356 \\
Th17/Treg & $0.05 \pm 0.03$ & $0.05 \pm 0.02$ & 0.838 \\
Tfh & $2.08 \pm 0.65$ & $2.06 \pm 0.59$ & 0.917 \\
Th1 & $0.14(0.03-0.24)$ & $0.20(0.05-0.30)$ & 0.204 \\
Th2 & $0.29(0.12-1.29)$ & $0.25(0.17-0.77)$ & 0.590 \\
Th1/Th2 & $0.25(0.09-1.19)$ & $1.18(1.10-1.50)$ & 0.449 \\
Breg & $5.68 \pm 2.79$ & $7.78 \pm 2.64$ & 0.033 \\
CD3 & $68.02 \pm 9.50$ & $66.60 \pm 11.37$ & 0.690 \\
CD4 & $32.71 \pm 7.03$ & $31.55 \pm 8.71$ & 0.666 \\
CD8 & $25.76 \pm 7.62$ & $26.92 \pm 10.20$ & 0.703 \\
CD4/CD8 & $1.40 \pm 0.56$ & $1.35 \pm 0.60$ & 0.799 \\
\hline
\end{tabular}

Data were shown as mean \pm SD (standard deviation) or median [Q25 - Q75] in AS patients is critical for clinicians to make adequate therapeutic decisions and provide personalized therapy for AS patients [5-27, 29-31]. Currently, there is still lack of definite predictors of response to anti-TNF- $\alpha$ therapy in AS patients. A recent systematic review and meta-analysis revealed that several clinical factors such as young age, male sex, and baseline BASDAI were predictors of better response to anti-TNF- $\alpha$ therapy in AS patients [28]. Serological markers such as baseline CRP and HLA-B27 were also identified as predictors of response to anti-TNF- $\alpha$ therapy in AS patients [28]. Nevertheless, the personalized therapy for AS patients is still difficult owing to the limited evidence from clinical studies or the lack of effective predictors [27, 32]. In this study, we assessed the roles of peripheral immunological profiles such as Th17, Treg, and Breg in predicting response to anti-TNF- $\alpha$ therapy of AS patients which are all HLA-B27 positive. We found that Breg was a possible predictor of response to anti-TNF- $\alpha$ therapy in AS patients, but the other immune cells such as Th17, Tfh, and Treg were not candidate predictor of response to anti-TNF- $\alpha$ therapy. The findings may be helpful to identify predictors of response to anti-TNF- $\alpha$ therapy and improve personalized therapy for AS patients from the perspective of immunological profiles in peripheral blood. 


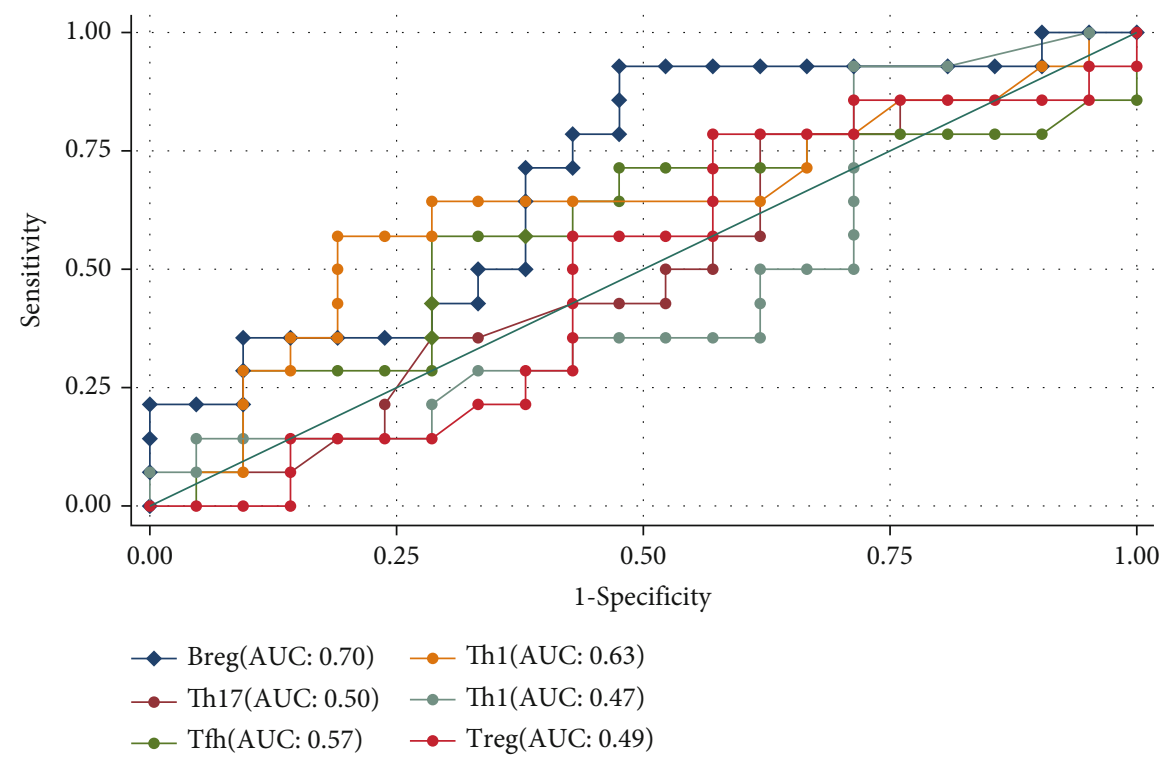

FIGURE 3: Assessment of the roles of circulating immune cells at baseline in predicting response to anti-TNF- $\alpha$ therapy in AS patients through ROC analysis (AUC: area under the ROC curve).

A major finding in our study is the potential role of Breg as a predictor of response to anti-TNF- $\alpha$ therapy in AS patients. Though anti-TNF- $\alpha$ therapy could increase the percentage of circulating Breg in AS patients, treatment nonresponders had a higher percentage of circulating Breg both at baseline and during follow-up, suggesting Breg as a possible predictor of response to anti-TNF- $\alpha$ therapy (Figure 2 and Tables 3 and 4). Several studies had assessed the changes of B cells with a regulatory phenotype in AS patients [21, 33, 34]. Cantaert et al. firstly reported that spondylarthritis patients had increased circulating $B$ cells with a regulatory phenotype $\left(\mathrm{CD} 19^{+} \mathrm{CD}^{+}\right)$[21]. A study by Bautista-Caro et al. also reported that AS patients had increased circulating $\mathrm{CD} 19^{+} \mathrm{CD} 24^{\text {hi }} \mathrm{CD} 38^{\text {hi }} \mathrm{B}$ cells with regulatory capacity, and anti-TNF- $\alpha$ therapy could significantly reduce the number of these B subset cells [34]. However, another study reported similar frequencies of $\mathrm{CD} 24^{+} \mathrm{CD} 38^{+}$B cells between AS patients and controls, but those cells from AS patients produced less IL-10 and thus had functional defects [33]. To our knowledge, apart from those 3 studies, no other study on the roles of Breg in AS has been published. Our study revealed that Breg was possibly related to response to antiTNF- $\alpha$ therapy in AS patients, and patients with high frequencies of Breg may predispose to poor response to antiTNF- $\alpha$ therapy, which provides new insights into the roles of Breg in AS. Currently, the molecular mechanism underlying the roles of Breg in the pathogenesis of AS is still unclear and needs to be elucidated in future studies.

While there are emerging data providing evidence for the involvement of $\mathrm{T}$ cell subsets in the pathogenesis of AS, few studies have evaluated the longitudinal changes of circulating immune cells after anti-TNF- $\alpha$ therapy in detail [35-37]. Additionally, our knowledge about their roles in predicting response to anti-TNF- $\alpha$ therapy in AS patients is still limited. The findings from our study confirmed the reduction in the frequencies of circulating lymphocyte sub- sets after anti-TNF- $\alpha$ therapy in AS patients. This study suggested that anti-TNF- $\alpha$ therapy could significantly and selectively reduce circulating proinflammatory immune cells such as Th17 and Tfh, but significantly increased the percentage of circulating Treg. While a large part of AS patients had gradual reductions in the percentage of $\mathrm{CD}^{+}$subsets such as Th17 and Tfh, some patients had increased percentages of circulating Th17 or Tfh after anti-TNF- $\alpha$ therapy, indicating the existence of variability in treatment response among AS patients. In addition, none of those $\mathrm{T}$ cell subsets were obviously related to response to anti-TNF- $\alpha$ therapy in AS patients (Figure 2 and Tables 3 and 4), suggesting that $\mathrm{T}$ cell subsets may have limited roles in predicting response to anti-TNF- $\alpha$ therapy in AS patients.

Our study suggested that anti-TNF- $\alpha$ therapy began to significantly increase the percentage of circulating Treg at 1 month after treatment $(P<0.005)$, and its mean percentage significantly increased from 5.62 to 8.06 after 6 months of anti-TNF- $\alpha$ therapy $(P<0.001$, Table 2$)$. It is uncertain whether the increase of Treg after anti-TNF therapy is responsible in part for the benefit of anti-TNF therapy in treating AS. A recent study suggested that expanding Treg through low-dose IL-2 was effective in treating AS [38]. The effect of anti-TNF- $\alpha$ therapy in treating AS may be at least partially mediated by its roles of increasing Treg cells, which need to be further studied.

This study used a prospective design and thus could provide a better assessment of the immunological changes in peripheral blood during anti-TNF- $\alpha$ therapy than those studies using retrospectively collected data. However, the findings in our study should be interpreted with caution because the sample size was not large enough. Besides, the treatment duration in this study was 6 months, which could not evaluate either the long-term efficacy of anti-TNF- $\alpha$ therapy or the long-term impact of anti-TNF- $\alpha$ therapy on immune cells. Further studies with larger number of AS 
patients and long-term follow-up are recommended to provide more evidence.

In summary, this study suggested that anti-TNF- $\alpha$ therapy could significantly reduce circulating proinflammatory immune cells such as Th17 and Tfh, but significantly increased the percentages of circulating Treg and Breg in AS patients. Moreover, circulating Breg may be a promising predictor of response to anti-TNF- $\alpha$ therapy in AS patients. Further prospective cohort studies with larger number of AS patients and long-term follow-up are warranted, and the molecular mechanism underlying the roles of Breg in the pathogenesis of AS needs to be elucidated.

\section{Data Availability}

All data are available upon request.

\section{Ethical Approval}

The study was approved by the ethics committee of The First Affiliated Hospital of Xiamen University.

\section{Consent}

Written informed consent was obtained from included patients.

\section{Conflicts of Interest}

All authors declare that they have no conflict of interest in this paper.

\section{Authors' Contributions}

Rongjuan Chen, Hongyan Qian, Xiaoqing Yuan, and Bin Wang made contributions to the study conception, performed the experiments and data collection, and wrote the manuscript. Shiju Chen, Yuan Liu, and Bin Wang analyzed the data. Bin Wang and Guixiu Shi critically reviewed the manuscript. Rongjuan Chen, Hongyan Qian, and Xiaoqing Yuan contributed equally to this work.

\section{Acknowledgments}

This work was supported by grants from the National Natural Science Foundation of China (Grant Nos. 81971536, 81971496, and No. U1605223).

\section{References}

[1] J. Sieper and D. Poddubnyy, "Axial spondyloarthritis," Lancet, vol. 390, no. 10089, pp. 73-84, 2017.

[2] J. W. Liew, S. Ramiro, and L. S. Gensler, "Cardiovascular morbidity and mortality in ankylosing spondylitis and psoriatic arthritis," Best Practice \& Research. Clinical Rheumatology, vol. 32, no. 3, pp. 369-389, 2018.

[3] K. L. Druce, L. Aikman, M. Dilleen, A. Burden, P. Szczypa, and N. Basu, "Fatigue independently predicts different work disability dimensions in etanercept-treated rheumatoid arthritis and ankylosing spondylitis patients," Arthritis Research \& Therapy, vol. 20, no. 1, p. 96, 2018.
[4] M. M. Ward, A. Deodhar, L. S. Gensler et al., "2019 update of the American College of Rheumatology/Spondylitis Association of America/Spondyloarthritis Research and Treatment Network recommendations for the treatment of ankylosing spondylitis and nonradiographic axial spondyloarthritis," Arthritis \& Rhematology, vol. 71, no. 10, pp. 1599-1613, 2019.

[5] H. Tahir, "Therapies in ankylosing spondylitis-from clinical trials to clinical practice," Rheumatology, vol. 57, suppl_6, pp. vi23-vi28, 2018.

[6] S. Dubash, C. Bridgewood, D. McGonagle, and H. MarzoOrtega, "The advent of IL-17A blockade in ankylosing spondylitis: secukinumab, ixekizumab and beyond," Expert Review of Clinical Immunology, vol. 15, no. 2, pp. 123-134, 2019.

[7] A. Deodhar, V. Strand, P. G. Conaghan et al., "Unmet needs in ankylosing spondylitis patients receiving tumour necrosis factor inhibitor therapy; results from a large multinational realworld study," BMC Rheumatology, vol. 4, no. 1, p. 19, 2020.

[8] L. J. Maxwell, J. Zochling, A. Boonen et al., "TNF-alpha inhibitors for ankylosing spondylitis," Cochrane Database of Systematic Reviews, vol. 4, article CD005468, 2015.

[9] U. Lindstrom, T. Olofsson, S. Wedren, I. Qirjazo, and J. Askling, "Biological treatment of ankylosing spondylitis: a nationwide study of treatment trajectories on a patient level in clinical practice," Arthritis Research \& Therapy, vol. 21, no. 1, p. 128, 2019.

[10] M. K. de Vries, E. V. Arkema, J. Jonsson et al., “Tuberculosis risk in ankylosing spondylitis, other spondyloarthritis, and psoriatic arthritis in Sweden: a population-based cohort study," Arthritis Care and Research, vol. 70, no. 10, pp. 1563-1567, 2018.

[11] EULAR, "EULAR RheumaMap: unmet needs in 2020," EULAR, vol. 2019, 2019.

[12] B. Noureldin and N. Barkham, "The current standard of care and the unmet needs for axial spondyloarthritis," Rheumatology, vol. 57, suppl_6, pp. vi10-vi17, 2018.

[13] H. Shen, J. C. Goodall, and J. S. Hill Gaston, "Frequency and phenotype of peripheral blood Th17 cells in ankylosing spondylitis and rheumatoid arthritis," Arthritis and Rheumatism, vol. 60, no. 6, pp. 1647-1656, 2009.

[14] L. Limon-Camacho, M. I. Vargas-Rojas, J. Vazquez-Mellado et al., "In vivo peripheral blood proinflammatory $\mathrm{T}$ cells in patients with ankylosing spondylitis," Journal of Rheumatology, vol. 39, no. 4, pp. 830-835, 2012.

[15] F. Xiao, H. Y. Zhang, Y. J. Liu, D. Zhao, Y. X. Shan, and Y. F. Jiang, "Higher frequency of peripheral blood interleukin 21 positive follicular helper $\mathrm{T}$ cells in patients with ankylosing spondylitis," Journal of Rheumatology, vol. 40, no. 12, pp. 2029-2037, 2013.

[16] E. Gracey, Y. Yao, B. Green et al., "Sexual dimorphism in the Th17 signature of ankylosing spondylitis," Arthritis \& Rhematology, vol. 68, no. 3, pp. 679-689, 2016.

[17] U. Syrbe, R. Scheer, P. Wu, and J. Sieper, "Differential synovial Th1 cell reactivity towards Escherichia coli antigens in patients with ankylosing spondylitis and rheumatoid arthritis," Annals of the Rheumatic Diseases, vol. 71, no. 9, pp. 1573-1576, 2012.

[18] S. Wu, T. Yang, F. Pan et al., "Increased frequency of circulating follicular helper T cells in patients with ankylosing spondylitis," Modern Rheumatology, vol. 25, no. 1, pp. 110-115, 2015.

[19] M. B. Bautista-Caro, I. Arroyo-Villa, C. Castillo-Gallego et al., "Decreased frequencies of circulating follicular helper $\mathrm{T}$ cell counterparts and plasmablasts in ankylosing spondylitis 
patients naïve for TNF blockers," PLoS One, vol. 9, no. 9, article e107086, 2014.

[20] S. Long, L. Ma, D. Wang, and X. Shang, "High frequency of circulating follicular helper T cells is correlated with B cell subtypes in patients with ankylosing spondylitis," Experimental and Therapeutic Medicine, vol. 15, no. 5, pp. 4578-4586, 2018.

[21] T. Cantaert, M. E. Doorenspleet, G. FrancoSalinas et al., "Increased numbers of CD5+ B lymphocytes with a regulatory phenotype in spondylarthritis," Arthritis and Rheumatism, vol. 64, no. 6, pp. 1859-1868, 2012.

[22] X. Y. Niu, H. Y. Zhang, Y. J. Liu, D. Zhao, Y. X. Shan, and Y. F. Jiang, "Peripheral B-cell activation and exhaustion markers in patients with ankylosing spondylitis," Life Sciences, vol. 93, no. 18-19, pp. 687-692, 2013.

[23] J. H. Vaile, J. B. Meddings, B. R. Yacyshyn, A. S. Russell, and W. P. Maksymowych, "Bowel permeability and CD45RO expression on circulating CD20+ B cells in patients with ankylosing spondylitis and their relatives," Journal of Rheumatology, vol. 26, pp. 128-135, 1999.

[24] P. Barbieri, I. Olivieri, G. Benedettini et al., "Polyclonal B cell activation in ankylosing spondylitis," Annals of the Rheumatic Diseases, vol. 49, no. 6, pp. 396-399, 1990.

[25] L. M. Ørnbjerg, C. H. Brahe, J. Askling et al., "Treatment response and drug retention rates in 24195 biologic-naïve patients with axial spondyloarthritis initiating TNFi treatment: routine care data from 12 registries in the EuroSpA collaboration," Annals of the Rheumatic Diseases, vol. 78, no. 11, pp. 1536-1544, 2019.

[26] G. J. Macfarlane, E. Pathan, G. T. Jones, and L. E. Dean, "Predicting response to anti-TNF $\alpha$ therapy among patients with axial spondyloarthritis (axSpA): results from BSRBR-AS," Rheumatology, vol. 59, no. 9, pp. 2481-2490, 2020.

[27] J. D. Taurog, A. Chhabra, and R. A. Colbert, "Ankylosing spondylitis and axial spondyloarthritis," The New England Journal of Medicine, vol. 374, no. 26, pp. 2563-2574, 2016.

[28] J. R. Maneiro, A. Souto, E. Salgado, A. Mera, and J. J. GomezReino, "Predictors of response to TNF antagonists in patients with ankylosing spondylitis and psoriatic arthritis: systematic review and meta-analysis," RMD Open, vol. 1, no. 1, article e000017, 2015.

[29] M. Lorenzin, A. Ortolan, P. Frallonardo, F. Oliviero, L. Punzi, and R. Ramonda, "Predictors of response and drug survival in ankylosing spondylitis patients treated with infliximab," $B M C$ Musculoskeletal Disorders, vol. 16, no. 1, p. 166, 2015.

[30] X. Baraliakos, A. S. Koenig, H. Jones, A. Szumski, D. Collier, and E. Bananis, "Predictors of clinical remission under antitumor necrosis factor treatment in patients with ankylosing spondylitis: pooled analysis from large randomized clinical trials," Journal of Rheumatology, vol. 42, no. 8, pp. 1418-1426, 2015.

[31] X. Baraliakos, A. Szumski, A. S. Koenig, and H. Jones, "The role of C-reactive protein as a predictor of treatment response in patients with ankylosing spondylitis," Seminars in Arthritis and Rheumatism, vol. 48, no. 6, pp. 997-1004, 2019.

[32] M. N. Magrey and M. A. Khan, "The paradox of bone formation and bone loss in ankylosing spondylitis: evolving new concepts of bone formation and future trends in management," Current Rheumatology Reports, vol. 19, no. 4, p. 17, 2017.
[33] M. Chen, L. Zhang, Y. Ren et al., "Defective function of $\mathrm{CD} 24^{+} \mathrm{CD} 38^{+}$regulatory B cells in ankylosing spondylitis," DNA and Cell Biology, vol. 35, no. 2, pp. 88-95, 2016.

[34] M. B. Bautista-Caro, E. de Miguel, D. Peiteado et al., "Increased frequency of circulating CD $19+\mathrm{CD} 24{ }^{\text {hi }} \mathrm{CD} 38^{\text {hi }} \mathrm{B}$ cells with regulatory capacity in patients with ankylosing spondylitis (AS) naïve for biological agents," PLoS One, vol. 12, no. 7, article e0180726, 2017.

[35] A. Taylan, I. Sari, D. L. Kozaci et al., "Evaluation of the T helper 17 axis in ankylosing spondylitis," Rheumatology International, vol. 32, no. 8, pp. 2511-2515, 2012.

[36] S. Dulic, Z. Vasarhelyi, A. Bajnok et al., "The impact of antiTNF therapy on CD4+ and CD8+ cell subsets in ankylosing spondylitis," Pathobiology, vol. 85, no. 3, pp. 201-210, 2018.

[37] F. M. Milanez, C. G. Saad, V. T. Viana et al., "IL-23/Th17 axis is not influenced by TNF-blocking agents in ankylosing spondylitis patients," Arthritis Research \& Therapy, vol. 18, no. 1, p. 52, 2016.

[38] M. Rosenzwajg, R. Lorenzon, P. Cacoub et al., "Immunological and clinical effects of low-dose interleukin-2 across 11 autoimmune diseases in a single, open clinical trial," Annals of the Rheumatic Diseases, vol. 78, no. 2, pp. 209-217, 2019. 